\title{
Mixed Cellularity Classic Hodgkin Lymphoma by Ann Arbor Stage
}

National Cancer Institute

\section{Source}

National Cancer Institute. Mixed Cellularity Classic Hodgkin Lymphoma by Ann Arbor

Stage. NCI Thesaurus. Code C141224.

An anatomic stage for mixed cellularity classic Hodgkin lymphoma based on the Ann Arbor classification criteria. 